\title{
First simultaneous observations of a near-relativistic electron spike event by both STEREO spacecraft
}

\author{
A. Klassen ${ }^{1}$, N. Dresing ${ }^{1}$, R. Gómez-Herrero ${ }^{2}$, and B. Heber ${ }^{1}$ \\ ${ }^{1}$ Institut für Experimentelle und Angewandte Physik, Universität Kiel, 24118 Kiel, Germany \\ e-mail: klassen@physik.uni-kiel.de \\ 2 SRG, Universidad de Alcalá, Dpto. de Física y Matemáticas, 28871 Alcalá de Henares, Spain
}

Received 20 January 2015 / Accepted 19 June 2015

\begin{abstract}
Solar electron spike events are a special subclass of near-relativistic electron events characterized by their short duration, symmetric time profile, and their strong anisotropic pitch angle distribution. All previous studied events were only observed by a single spacecraft (s/c). For the first time, we present measurements of this kind of an electron spike event that was observed simultaneously by both close-spaced STEREO s/c on 2 May 2014. The longitudinal separation angle between STEREO-A (STA) and STEREO-B (STB) was $38^{\circ}$. The spikes at STA and STB are characterized by very short durations of $\leq 12 \mathrm{~min}$ at full-width at half maximum (FWHM) and almost identical and symmetric time profiles. They exhibit similar properties in durations, pitch angle distributions (PADs), energy spectra, and peak intensities. The spike parent source was situated close to the STA nominal magnetic footpoint and was separated by $48^{\circ}$ from STB's footpoint. The intensity distribution and the relative onset timing behaved opposite to what is expected: STB measured a higher intensity and an earlier onset than the nominally better connected STA. We suggest that the spike electrons undergo a substantial nonradial injection into interplanetary (IP) space from the parent source, a flaring active region (AR), and propagate in a strong nonradial diverging magnetic field. In addition we show that because of the spike properties it is evident that, irrespective of how the electrons were injected into the IP medium, the en route particle scattering conditions along the paths to STA and STB were very similar. Evidently both s/c detected different parts of the same beam whose angular extension was at least $38^{\circ}$ at $1 \mathrm{AU}$, comparable to the longitudinal separation between STA and STB.
\end{abstract}

Key words. Sun: flares - Sun: particle emission - acceleration of particles - interplanetary medium

\section{Introduction and motivation}

Solar electron spike events (Haggerty \& Roelof 2009; Klassen et al. 2011, 2012) represent a special subclass of solar energetic particle (SEP) events and were only detected until now by single spacecraft (s/c). In comparison to normal impulsive events, they show a substantially shorter duration of $\leq 20$ min at fullwidth at half maximum (FWHM), nearly symmetric intensity time profiles and narrower pitch angle distribution (PAD) during the whole spike duration, suggesting very weak scattering along the propagation path from the source to the s/c. Almost all spikes show a close, one-to-one time correlation with type III radio bursts and EUV coronal jets (Klassen et al. 2011), indicating that all three phenomena are likely produced by the same driver (Klassen et al. 2012).

During 2014 both STEREO s/c approached their superior solar conjunctions (to occur in 2015) and the separation angle between both $\mathrm{s} / \mathrm{c}$ was less than $40^{\circ}$. This unique constellation allows us to study the particle intensity distribution at relatively small separation distances.

In contrast to so-called widespread SEP events (e.g., Dresing et al. 2012; Lario et al. 2014; Richardson et al. 2014; Gómez-Herrero et al. 2015) showing a very broad up to $360^{\circ}$ circumsolar particle distributions at $1 \mathrm{AU}$, the solar electron spike events represent the opposite extreme of events that have a rather narrow spatial particle distribution, as suggested by the fact that, until now, all detected spikes were only observed by a single s/c.
In this paper, we study the first electron spike observed simultaneously by STEREO-A (STA) and STEREO-B (STB), on 2 May 2014. The intensity and anisotropy evolution, the energy spectra, and the onset timing in relation to the observed type III radio bursts are interpreted as particles arriving from the same active region released at the Sun by the same activity phenomena. We show that the electron spike event had an unexpected intensity distribution and onset timing, and provides an opportunity to investigate the magnetic connectivity between the parent source and different $\mathrm{s} / \mathrm{c}$. We investigate why the nominal Parker spiral is not able to describe the electron propagation path from the Sun to both s/c and how different injection models can explain the observations.

\section{Instrumentation}

The electron observations are provided by the identical Solar Electron and Proton Telescopes (SEPT) on board both STEREO s/c. The "SEPT consists of two dual double-ended magnet/foil particle telescopes, which cleanly separate and measure electrons in the energy range from $30-400 \mathrm{keV}$ and protons from 60 $7000 \mathrm{keV}$. Anisotropy information on a non-spinning s/c is provided by the two separate telescopes: SEPT-E looking in the ecliptic plane along the Parker spiral magnetic field both towards and away from the Sun, and SEPT-NS looking vertical to the ecliptic plane towards North and South" (Müller-Mellin et al. 2008). Additionally, we use PLASTIC solar wind data 


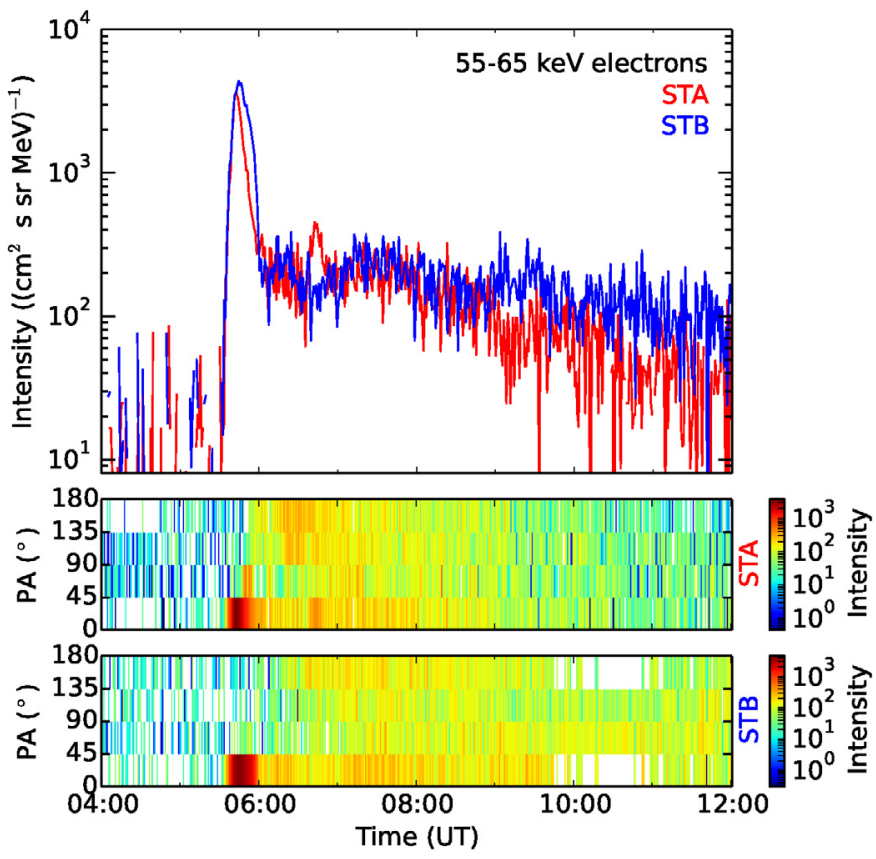

Fig. 1. Electron spike on 2 May 2014. Top: spike intensity time profiles detected at STEREO-A (red) and STEREO-B (blue) with SEPT-Sun telescope viewing in the sunward direction, showing very similar spike + ramp shapes in the energy range of 55-65 keV. STEREO-B measured a higher intensity and an earlier onset compared to STEREO-A. Bottom: intensity pitch angle distribution divided in four bins ( $=4$ telescopes). White intervals denote sectors with no pitch angle coverage.

(Galvin et al. 2008), SWAVES radio emission spectra (Bougeret et al. 2008), EUV images from SECCHI-EUVI (Howard et al. 2008), and MAG data (Acuña et al. 2008) from both STEREO s/c.

\section{Observations}

The SEP event on 2 May 2014 starting at 05:32 UT (Fig. 1) is the first case of 90 electron spikes that was observed simultaneously by both STEREO s/c since the beginning of the STEREO mission in 2006 and was not detected by s/c close to Earth. At that time the longitudinal separation between both STEREOs was $38^{\circ}$ and the calculated nominal Parker spiral footpoint separation was $47^{\circ}$ (Figs. 2 and 6) when using the measured solar wind speeds (Fig. 7). The difference in orbital and magnetic footpoint separation results from the different solar wind speeds as measured by STA and STB (360 and $338 \mathrm{~km} \mathrm{~s}^{-1}$, respectively) and the different radial distances to the Sun of 0.96 and $1.02 \mathrm{AU}$ for STA and STB, respectively.

The spike was coincident with a mixed solar activity (see Fig. 3) including a small flare $(\mathrm{F})$, a jet $(\mathrm{J})$ and brightening loops (L) in two neighboring active regions (ARs) situated $10^{\circ}$ apart from each other in latitude. A more precise identification of the parent source of the spike event (flare, jet or brightening loops) was not possible because of a nearly simultaneous occurrence of all phenomena and the low 5-min cadence of the EUVI images.

Nevertheless, we suggest that the flare $(F)$ was the most likely source since the flare start time is in better agreement with the onset of the type III radio burst starting at 05:09 UT (Fig. 4). We exclude the jet to be the source because a number of recurrent jets were observed from the same region and they were neither

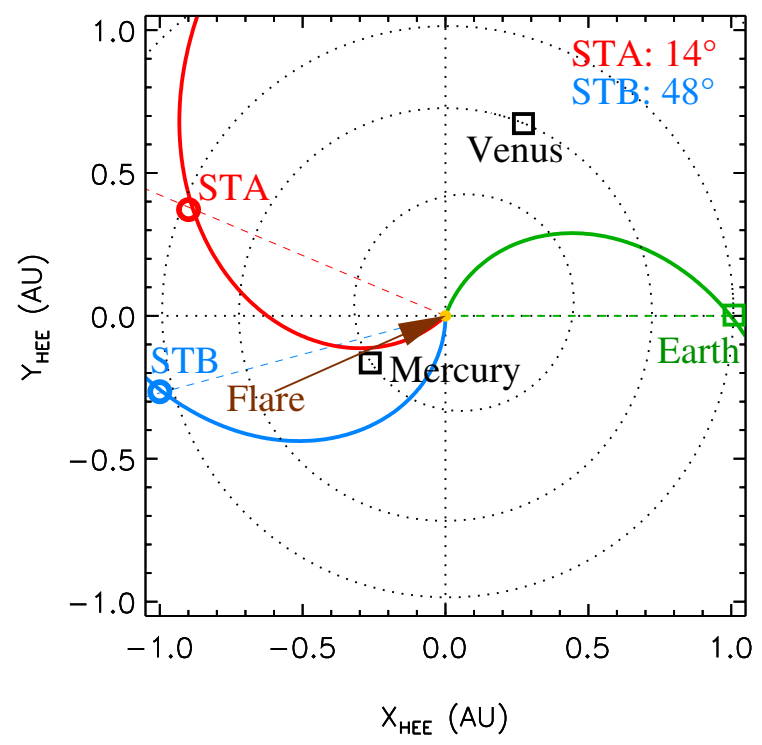

Fig. 2. Longitudinal positions of STEREO-A, STEREO-B, and Earth and the nominal Parker spirals connecting them to the Sun. The brown arrow marks the location of the parent flare. The angular separations between the s/c nominal magnetic footpoints and the position of the parent flare ((F) in Fig. 3) are inserted in the upper right corner.

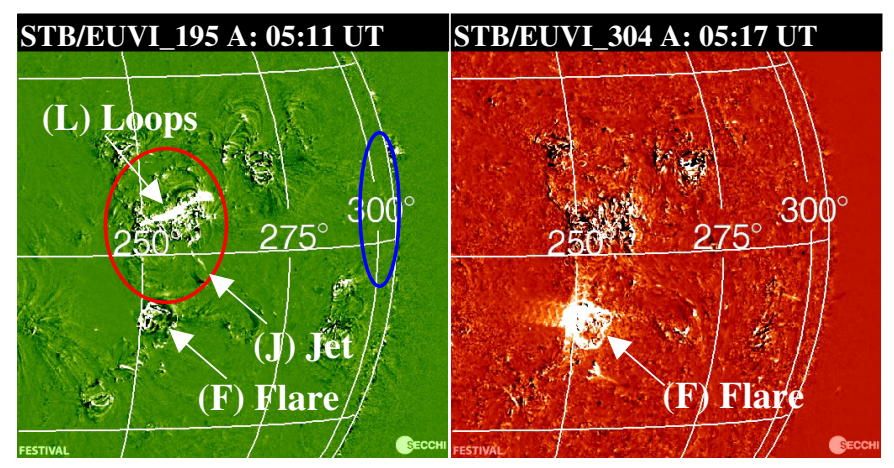

Fig. 3. STEREO-B EUVI $195 \AA$ (left) and $304 \AA$ (right) difference images showing the flare $(\mathrm{F})$, the jet $(\mathrm{J})$ and brightening loops $(\mathrm{L})$ associated with the spike on 2 May 2014. The images were taken during the onset (left) and the end (right) of type III bursts (Fig. 4), respectively. STEREO-A and STEREO-B magnetic footpoints are marked by red and blue ellipses, respectively.

associated with type III radio bursts nor with in situ electron events. The loop system (L, Fig. 3) begin to brighten $\leq 10 \mathrm{~min}$ before the type III bursts onset suggesting that they are less favorable candidate for the source of the particle injection.

However, for our analysis, it is less important which of the mentioned phenomena was ultimately responsible for the particle injection. More essential is that they all occur close to each other and close to the expected nominal magnetic footpoint of STA, shown by the red ellipse in Figs. 3 and 6. The ellipses represent the $10^{\circ}$ uncertainty for the backmapping procedure as assumed by e.g., Ippolito et al. (2005) and Klein et al. (2008). The backmapped latitude is assumed to be the projected latitude of the s/c. Indeed, the STA angular magnetic footpoint separations with respect to the loops, to the jet, and to the flare were $0^{\circ}, 4^{\circ}$, and $14^{\circ}$, respectively. For STB, these separation were much larger: $40^{\circ}, 42^{\circ}$, and $48^{\circ}$, respectively. In addition the spike was associated with a narrow coronal mass ejection $(\mathrm{CME})$ (width $\leq 76^{\circ}$, speed $\leq 781 \mathrm{~km} \mathrm{~s}^{-1}$; 
Table 1. Event properties.

\begin{tabular}{|c|c|c|}
\hline Properties/spacecraft & STA & STB \\
\hline Spike onset, UT & 0533 & 0532 \\
\hline - peak intensity, particles/( $\mathrm{cm}^{2} \mathrm{~s}$ sr MeV) & 3640 & 4400 \\
\hline - time to maximum, min & 8 & 11 \\
\hline - spectral index $(45-295 \mathrm{keV}), \gamma$ & $-3.73 \pm 0.09$ & $-3.96 \pm 0.07$ \\
\hline - energy range, $\mathrm{keV}$ & $\leq 295$ & $\leq 295$ \\
\hline Type III bursts onset (SWAVES), UT & 0509 & 0509 \\
\hline Local Langmuir waves & yes & yes \\
\hline Onset delay $^{a}$, spike vs. type III onset, min & 10.6 & 8.4 \\
\hline Mag. footpoints separation to loops/jet/flare, $\left(^{\circ}\right)$ & $0 / 4 / 14$ & $40 / 42 / 48$ \\
\hline Flare Carrington longitude; latitude, $\left(^{\circ}\right)$ & $252 ;-8$ & $252 ;-8$ \\
\hline Solar wind speed $V_{\mathrm{sw}}, \mathrm{km} \mathrm{s}^{-1}$ & 360 & 338 \\
\hline CME: speed, $\mathrm{km} \mathrm{s}^{-1}$; angular width ${ }^{b},\left(^{\circ}\right)$ & $781 ; 56$ & $735 ; 76$ \\
\hline Type II burst (SWAVES/ground-based) & no & no \\
\hline EIT-wave & no & no \\
\hline
\end{tabular}

Notes. All spike parameters were derived for electrons in the energy range $55-65 \mathrm{keV}$. ${ }^{(a)}$ In solar release time (SRT): taking into account the measured solar wind speed $V_{\mathrm{sw}}$, the travel time of radio emission and electrons from the Sun to the s/c, and the s/c distance from the Sun (0.96 and $1.02 \mathrm{AU}$ for STA and STB, respectively). ${ }^{(b)}$ CME parameters taken from CACTUS (http://sidc.oma. be/cactus/; Robbrecht \& Berghmans 2004).

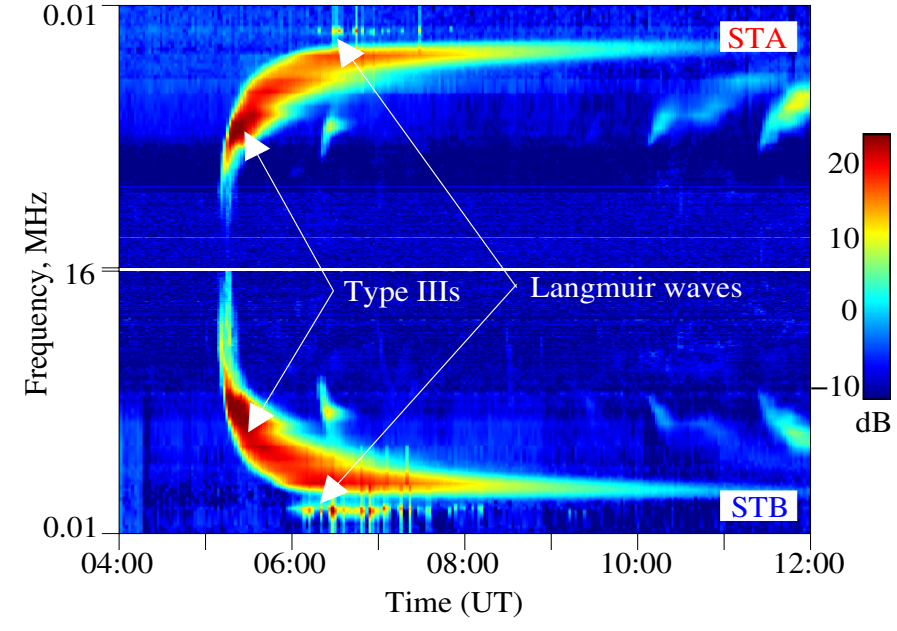

Fig. 4. Dynamic radio spectra of the interplanetary type III radio bursts occurring between 05:09-05:17 UT on 2 May 2014 around $16 \mathrm{MHz}$ and the local Langmuir waves associated with the spike event (adapted from: http://swaves.gsfc.nasa.gov/cgi-bin/wimp.py, courtesy SWAVES team).

CACTUS $^{1}$ ) as observed at COR2/STA/STB, with interplanetary (IP) type III radio bursts and locally generated Langmuir waves, identified as bursty patches at the low edge of type IIIs near to $20 \mathrm{kHz}$ by both STEREO (Fig. 4). The Langmuir waves are usually interpreted as plasma waves locally generated by the arrival of low-energy electrons $(\leq 2-20 \mathrm{keV})$ primarily responsible for the type III radio emission (e.g., Ergun et al. 1998; Klassen et al. 2012). The spike event and the parent EUV activity were not accompanied by an EIT-wave and type II radio burst.

Figure 1 presents the electron time profiles in the energy range of 55-65 keV as measured by the sunward pointing telescopes at STA and STB, showing very similar shapes typical for so-called spike events (e.g., Klassen et al. 2012, and references therein). The symmetric intensity profiles with a prompt onset, a prompt decay, and short durations of 8 and 12 min at FWHM for STA and STB, respectively, are followed by a prolonged ramp

\footnotetext{
1 http://secchi.nrl.navy.mil/cactus/index.php? p=SECCHI $-\mathrm{A} / 2014 / 05 /$ out $/$ CMEO010/CME . html
}

of substantial lower intensity and more isotropic fluxes. Strong anisotropic PADs presented in Fig. 1, together with the clear velocity dispersion (not shown here), suggest that both s/c detected practically an identical beam streaming from the Sun at PA $0^{\circ}$. This PA corresponds to the outward magnetic field directed away from the Sun. The outward field is also consistent with the positive polarity of the open solar magnetic field lines in the vicinity of the s/c connection points (Fig. 6).

The PADs of the ramp contained a bidirectional component of enhanced flux at pitch angle around $0^{\circ}$ and $180^{\circ}$ suggesting that the spike electrons possible bouncing between strong scattering regions (mirrors) situated beyond the s/c orbits and somewhere between the Sun and s/c (e.g., Tan et al. 2009; Klassen et al. 2012). An additional small spike-like event only detected by STA at 06:38 UT was temporally associated with a low frequency type III around 06:20 UT (Fig. 4). No clear associated solar activity was found for this time interval. Further parameters of the spikes (Table 1) confirm the similarity of the observed beams at STA and STB: the peak intensities of 3640 and 4400 particles $/\left(\mathrm{cm}^{2} \mathrm{~s}\right.$ sr MeV), respectively, the time from spike onset to maximum (STA: $8 \mathrm{~min}$; STB: $11 \mathrm{~min}$ ), and the peak intensity spectra with power-law index of $\gamma_{\mathrm{STA}}=$ $-3.73 \pm 0.09$ and $\gamma_{\mathrm{STB}}=-3.96 \pm 0.07$ in the energy range from 45 to $295 \mathrm{keV}$; Fig. 5 . Figure 6 presents the synoptic map from the Global Oscillation Network Group (GONG) with open field lines connecting to the ecliptic plane computed using the Potential Field Source Surface (PFSS) model. Red denotes negative polarity and green positive polarity. The positions of parent source candidates are marked by gold diamonds: F denotes flare, J denotes the small EUV jet, and L denotes brightening loops. The heliospheric current sheet separating opposite magnetic field polarities is marked by the white wavy line and the hatched areas correspond to coronal holes.

As seen in Fig. 2, the MESSENGER s/c orbiting Mercury was favorably located between of STA and STB to be able to detect the spike. Unfortunately, the EPS particle instrument did not observe any flux enhancements during the time of the spike, mainly because of the high instrumental background and because the FOV of EPS was directed toward Mercury, i.e., away from the Sun (D. Lario, priv. comm.). 


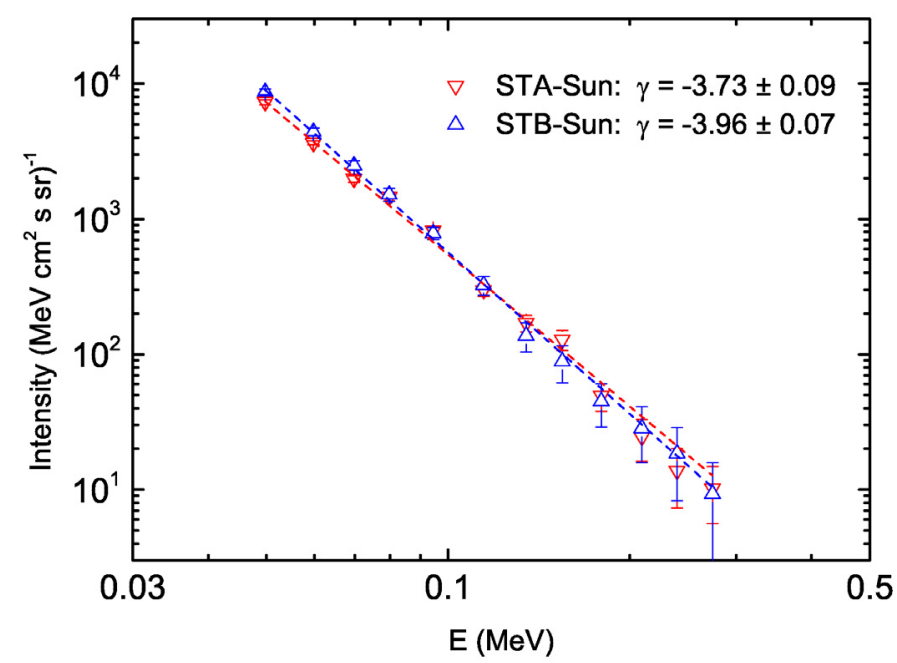

Fig. 5. Derived electron peak intensity spectra in energy range 45-295 keV with subtracted pre-event background spectra. The red and blue straight lines are the power-law fits to the STEREO-A and STEREO-B data, showing very similar slopes.

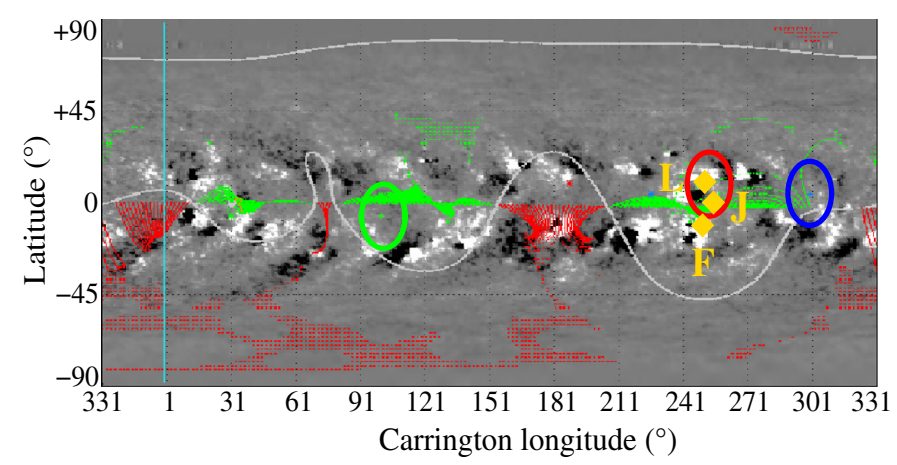

Fig. 6. Synoptic magnetic field map from the Global Oscillation Network Group (GONG) showing open magnetic field lines connecting to the ecliptic plane. The green lines show the outward and red the inward magnetic fields, respectively. s/c estimated nominal magnetic footpoints along the Parker spiral are indicated by ellipses (STEREO-A in red, STEREO-B in blue and Earth in green). The positions of parent source candidates are marked by gold diamonds: $\mathrm{F}$ - flare, $\mathrm{J}$ - the small EUV jet, L - brightening loops.

\subsection{In situ plasma and magnetic field observations and interplanetary conditions}

Figure 7 shows an overview of in situ plasma and magnetic field data as observed by STA and STB from May 1 to May 6. The panels display from top to bottom the solar wind proton speed, density, temperature, magnetic field magnitude, its latitudinal angle, and its azimuthal angle in the RTN coordinate system. The vertical line marks the electron spike onset time. When the SEP event started, both STB and STA were in regions of positive magnetic field polarity. As discussed above the observed IP environment implies quiet conditions during the whole period. Indeed, both s/c were embedded in slow solar wind streams $\left(V_{\mathrm{sw}}<360 \mathrm{~km} \mathrm{~s}^{-1}\right)$ and in smooth magnetic fields staying in the same sector with positive polarity. The main difference was a weak corotating interaction region likely observed by STB, indicated by the enhancement in magnetic field, density, and kinetic temperature (but with a very modest solar wind speed increase). Nevertheless, the key parameter for the magnetic field backmapping is the solar wind speed, which is very similar at

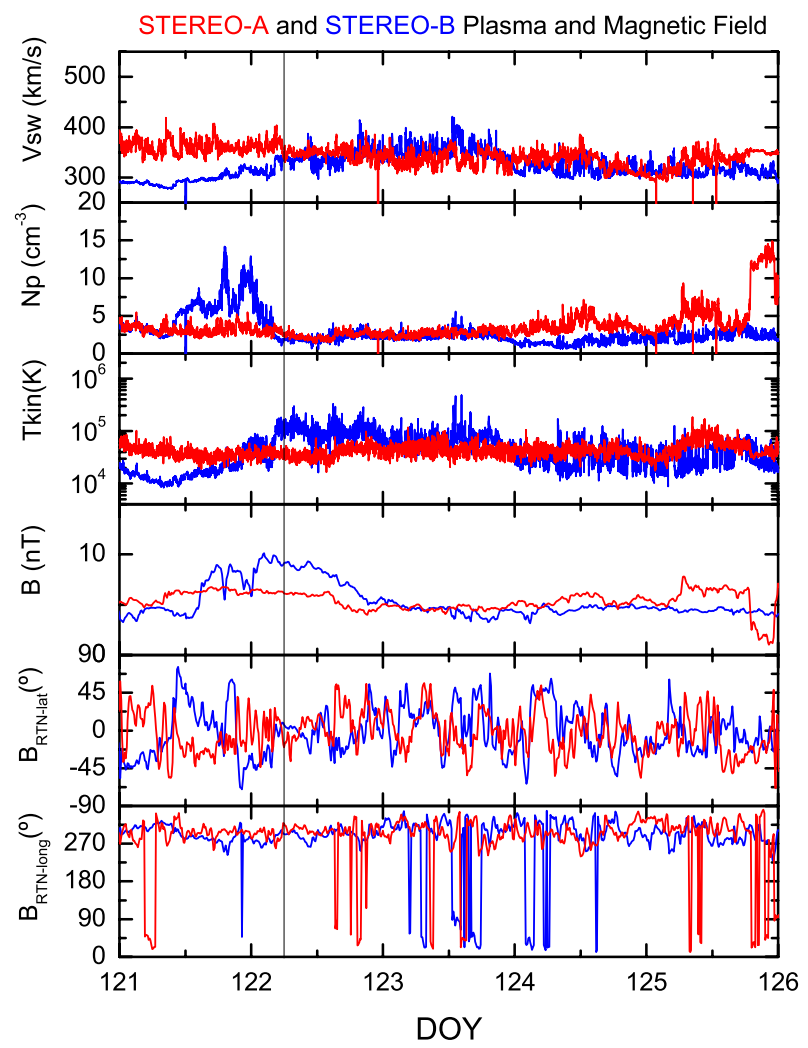

Fig. 7. Solar wind and magnetic field measurements at STEREO-A (red) and STEREO-B (blue) from 1 May to 6 May 2014. From top to bottom: solar wind proton speed, proton density, proton temperature, magnetic field magnitude, magnetic field latitudinal, and longitudinal angles. Vertical line marks the spike time.

both s/c during and after the particle event. No shocks and disturbances were observed by STEREO or by SOHO/ACE, which confirms the suggestion that there were no strong and fast CMEs and interplanetary coronal mass ejections (ICMEs) during the whole time period presented in Fig. 7.

The simultaneous detection of similar spikes suggest that both STEREO s/c were similarly very well connected to the particle source even though the nominal connecting footpoints were separated by $\sim 48^{\circ}$. A possible explanation is that field lines passing STB were deflected to the east by $48^{\circ}$ between the Sun and the s/c because of high solar wind speed. To get this kind of a shift in connection, a high solar wind speed ( $>900 \mathrm{~km} \mathrm{~s}^{-1}$ ) would be required along a major part of the particle propagation path. However, there is no observational evidence for these high solar wind speeds. The measured solar wind speed was slow at both $\mathrm{s} / \mathrm{c}$, and in particular was $\leq 360 \mathrm{~km} \mathrm{~s}^{-1}$ at STB during and also a few days after the spike event (Fig. 7).

An en route CME/ICME ejected before the SEP event may, in principle, be able to modify the structure of the IP magnetic field between the s/c and the Sun and could shift the connecting footpoints from the nominal position. For instance, a CME occurring close to the STB nominal footpoint could displace the connection point eastward toward the location of the parent flare. However, examining STEREO and SOHO coronagraph observations, we found no large/fast CMEs occurring during the few days prior to the SEP event that might have modified the s/c connection. In particular, we checked the CME lists and movies, especially the east CMEs as seen from Earth/SOHO and the west CMEs as seen from STA. The absence of in situ IP shocks at least during four days after the SEP event supports the suggestion that 
the IP conditions did not essentially change the s/c connections (see Fig. 7). Therefore, it is reasonable to assume that the large magnetic field spread or nonradial field distribution takes place below the source surface, providing a close connection to the parent flare for STB as well as for STA.

\subsection{Time delays}

Although the shapes of the spike time profiles at both $\mathrm{s} / \mathrm{c}$ are very similar, both spikes were delayed relative to the type III onsets and with respect to each other. Indeed, the spike onsets at STA and STB were delayed by 10.6 and 8.4 min relative to type III onsets, respectively. In these calculations, in order to compare the remote sensing and in situ data, we used the solar release time of the spike in the energy range of 55-65 keV and type III onsets taking the nominal Parker spiral length into account. While the latter depends on the solar wind speed measured during the spike and on the radial distance of the $\mathrm{s} / \mathrm{c}$ from the Sun, which is slightly different for both $\mathrm{s} / \mathrm{c}$, the different radio wave travel times to STA and STB only depend on the distance from the Sun.

In the corresponding calculations we assume that the spike electrons injection coincides with the decametric-hectometric (DH: 1-16 MHz, as observed by SWAVES) type III onset (e.g., Cane 2003; Kahler et al. 2007) and that both the low-energy electrons responsible for type III generation and the presented nearrelativistic electrons belong to the same particle population.

STA, which is the s/c closest to the Sun (0.96 AU) and best connected to the flare, shows a lower peak intensity and a slightly later onset (1 $\mathrm{min})$ relative to $\mathrm{STB}$, which is further away from the Sun (1.02 AU). If we adjust the STB distance to $0.96 \mathrm{AU}$ as for STA then the relative onset delay between both would be longer than $2 \mathrm{~min}$.

The spike onset delays relative to type III burst onset of 10.6 and 8.4 min imply a substantially larger propagation path from the Sun to STA and STB than their paths along the nominal Parker spiral of $\sim 1.15$ and 1.23 AU. These "effective" propagation paths are very similar for both s/c and range between 1.71-1.69 AU for STA and STB, respectively. This result is in agreement with values found in previous investigations, suggesting an essentially larger propagation path from the Sun to $1 \mathrm{AU}$ for electrons during impulsive events (e.g., Ragot 2006; Ragot \& Kahler 2008; Kahler et al. 2011).

Alternatively, if we assume a delayed injection of near relativistic electrons into the IP medium relative to the type IIIs (e.g., Wang et al. 2006) and suppose that the electrons arriving at both STEREO s/c belong to the same population and are injected at the same time, then the earlier onset at STB would still imply that the connection path length to STB is shorter than to STA. A third possibility is that the electrons injected onto field lines connecting to STB earlier than onto field lines connecting with STA.

\subsection{Summary of observational results}

The studied short-lived SEP event was the first electron spike detected simultaneously at both STEREO s/c as they were separated from each other by $38^{\circ}$ in longitude and it shows an unexpected intensity ratio and an unexpected onset timing. The observational results can be summarized as follows:

1. The spike's intensity and anisotropy time profiles, as well as the peak energy spectra are nearly identical for STA and STB, indicating a common source.

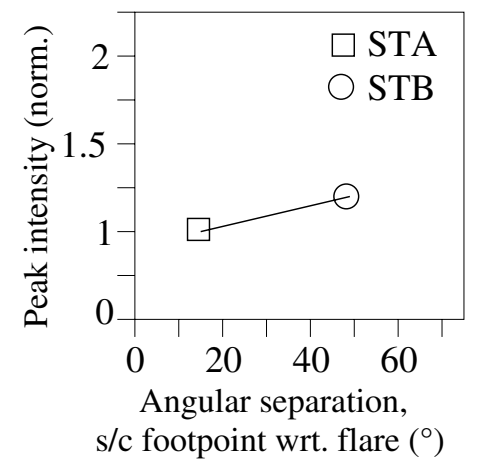

Fig. 8. Electron peak intensities with respect to (wrt) angular separation between the flare and the s/c magnetic field footpoint. STEREO-B detected first the spike onset. The peak intensities are normalized to spike maximum measured at STEREO-A. The pre-event background is subtracted.

2. Nonetheless, there is still a small relative difference in the peak intensities (factor $\leq 1.2$ ) as measured by STA and STB. The STB had a larger nominal footpoint separation from the source region, but measured a higher intensity than the closer connected STA. At the same time the magnetic separation angle between the s/c and the parent flare was $48^{\circ}$ and $14^{\circ}$ for STB and STA, respectively, and the calculated magnetic footpoint angular separation between both s/c was $47^{\circ}$.

3. The spikes show an unexpected onset timing, i.e., the nominally better connected STA detected a later onset than the farther separated STB with an adjusted time delay of $2 \mathrm{~min}$.

4. We find a significant spike time delay with respect to the type III radio burst onset of $10.6 \mathrm{~min}$ for STA and $8.4 \mathrm{~min}$ for STB. It means that the electrons propagate along nonnominal Parker spirals and the true propagation path to both $\mathrm{s} / \mathrm{c}$ of $\sim 1.7 \mathrm{AU}$ is essentially longer than the length of the nominal Parker spiral (1.15-1.23 AU) calculated using the measured solar wind speeds.

5. The spike short durations and strong anisotropies suggests that, irrespectively of how and when the electrons were injected into the IP medium, the particle scattering conditions along the paths to STA and STB were very similar.

6. Evidently both s/c detected different parts of the same beam, whose angular broadness was $\geq 38^{\circ}$ at $1 \mathrm{AU}$, corresponding to the longitudinal separation between STA and STB.

\section{Discussion}

The special properties of short duration and strongly anisotropic electron spikes in general, and especially of the spike on 2 May 2014, pose two questions: How wide is the real spatial extension of a spike beam at 1 AU? How are the electrons injected into the IP medium forming the measured nonexpected spatial angular distribution of intensity peaks shown in Fig. 8? The first question is particularly intriguing in context of the rareness of simultaneous multispacecraft spike observations implying a rather narrow beam size below $50^{\circ}$. Indeed, the multispacecraft electron spike event presented here is the first of more than 90 spikes detected at both STEREO s/c from 2010 till October 2014. Furthermore, it is the first from 27 events in 2014, when the separation angle between STA and STB was relatively small ranging between 51 and $33^{\circ}$.

The longitudinal angular separation between STA and STB was $38^{\circ}$. Therefore it is evident that the observed electron beam was extended over at least $38^{\circ}$ at $1 \mathrm{AU}$. The extension was probably substantially larger because the detected peak intensities at 

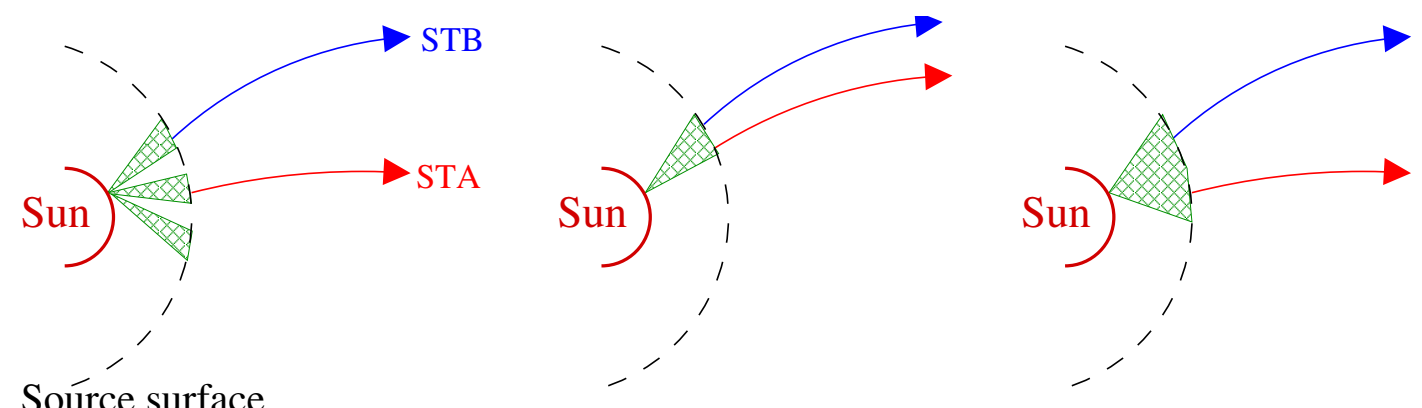

Fig. 9. Sketch illustrating the suggested injection scenarios and electron source(s) distribution at the source surface (not drawn to scale). Left: multipoint source; middle: single point-like source; and right: extended source. The green hatched cone(s) shows the beam(s) injected from the parent source.

both s/c were very comparable as shown in Figs. 1 and 8, implying that the intensity could not also substantially decrease outside of this angle and that the real beam might have a spread larger than $38^{\circ}$.

Figure 8 presents the spike peak intensities with respect to the angular separations between the s/c footpoints and the flare. The peak intensities are normalized to the spike maximum at STA and the pre-event intensity background is subtracted. On the one hand, two available points do not allow us to provide a fit and determine the detailed shape of the angular distribution. On the other hand, propagation models assume (e.g., Strauss \& Fichtner 2015) that if the particles stream along the nominal Parker spiral then the measured distribution would be symmetrical Gaussian with a maximum at the angle $0^{\circ}$, i.e., at the angle with the best magnetic connection to the flare. In this case, it is obvious that no Gaussian distributions with a maximum around $0^{\circ}$ can fit the peak intensity points in Fig. 8. If the Gaussian is shifted to the right (in our case to the west) with a maximum around $30^{\circ}$, however, then a narrow and nearly symmetrical Gaussian could fit the both peaks. However a displaced, narrow Gaussian that happens to give approximately similar intensities at each $\mathrm{s} / \mathrm{c}$ is a rather contrived scenario. Therefore, to explain the intensity peak position relative to the optimal connection to the flare $\left(0^{\circ}\right.$ in Fig. 8), an another scenario that might help to account for the observations is to assume that the electrons were injected onto nonradial diverging coronal magnetic field lines tilted toward the magnetic footpoint of STB and/or away from the footpoint of STA.

This assumption cannot be proven because of the lack of photospheric magnetic field data (and its distribution) for the backside of the Sun as seen from Earth and a lack of radio imaging observations for this time period, which could provide the trajectory of the type III burst sources and their nonradial propagation. Nevertheless, there are strong hints from previous studies inferring that this specific scenario may be plausible. In fact, nonradial particle injections during impulsive events, in general (e.g., Klein et al. 2008; Klassen et al. 1999, 2003), and during spike events, in particular (Klassen et al. 2012), often occur in association with flares and type III bursts.

How particle injection influences the intensity distribution at $1 \mathrm{AU}$ seems to be quite complicated and may be explained by at least three scenarios regarding the source size. Figure 9 sketches three simplified scenarios of different beam structures below the source surface (green hatched cone) together with the $\mathrm{s} / \mathrm{c}$ connections to the source(s). Here we approximate the acceleration source itself (flare/jet) in the low corona as a point source.

1. Multipoint source at the source surface (Fig. 9 left). The fact that spikes usually appear to have extensions of $\leq 50^{\circ}$ suggests a scenario with multipoint injection in which particles from a single acceleration source are injected into different coronal magnetic structures below the source surface appearing as several distinct spatially separated regions at the source surface. In this scenario, both STEREO s/c are connected to different regions at the source surface. However, it is unclear in this scenario why the spike event properties at the two s/c are very similar. Because of the limited longitudinal observations by the two STEREO s/c at $1 \mathrm{AU}$ there is no proof that the electron spike event would have been measured at a point between the two s/c. If a s/c had been situated between the STEREOs, it would have observed a different intensity. This intensity might be lower if connected to the source surface to a region that is not injecting particles.

2. Single point-like source at the source surface (Fig. 9 middle). The particles are injected from the parent source into a single flux tube connecting the flare to the Parker spirals at the source surface. From these the magnetic field strongly diverges mainly toward STB and toward STA. Consequently, both s/c would detect similar spike events with simultaneous onsets. In this case, however, the fields connecting the footpoints of both STA and STB are situated close to each other. This means that some structure or mechanism is required to distort the IP field lines and bring their footpoints closer together. A transient solar wind structure might be involved distorting the nominal Parker spiral, but as discussed in Sects. 3 and 3.1, there is no compelling evidence that this kind of structure was present.

3. Extended source at the source surface (Fig. 9 right). The particles propagate away from the flare along fan-shaped magnetic field lines (e.g., Klein et al. 2008), which at the source surface extend over at least the $47^{\circ}$, i.e., approximately equal to the nominal footpoint separation between STA and STB. In this case, the footpoint locations of both s/c and the magnetic field distribution shown in Fig. 6 are correct, but it is not evident why STB, the worse connected s/c, detected an earlier spike onset and a higher intensity. Furthermore, similar peak intensities observed at both s/c at $1 \mathrm{AU}$ also require a similar intensity distribution in an extended injection source. This kind of uniform distribution close to the parent source contradicts the radio observations of coronal and IP type III sources showing normally rather a Gaussian spatial intensity distribution (Raoult \& Pick 1980; Fitzenreiter et al. 1977).

For all scenarios, the problem of the large spike onset delay relative to the type III burst onset is still existent and relevant, and up to now not really understood. It may be solved assuming an essentially longer electron propagation path in comparison to the nominal Parker spiral connecting the source close to the Sun with the s/c (Ragot 2006). 


\section{Summary}

For the first time, both STA and STB s/c simultaneously observed the same electron spike event demonstrating very similar properties in intensities, PADs, and energy spectra, implying the observation of the same single electron beam originating from a small solar EUV/flare activity accompanied by type III radio bursts and a narrow $\mathrm{CME}$. The spike beam angular extension was larger than $38^{\circ}$ at $1 \mathrm{AU}$ and it was injected from the parent AR into the IP medium rather strongly nonradial towards STB along a tilted and diverging magnetic field in the low corona.

We discussed three possible scenarios that might be able to explain some of the observed unexpected features. While scenario (1) with multiple channels connecting the flare with the source surface shows difficulties in explaining the comparable spike intensities at both $\mathrm{s} / \mathrm{c}$ as well as the spike onset timing, scenario (2) based on disturbed magnetic connections in the IP medium is not supported by the in situ plasma and magnetic field observation. Therefore, we favor scenario (3), which is based on an extended parent source situated in diverging and rather nonradial magnetic fields in the corona along which the spike electrons escape into the IP medium toward both STEREO s/c.

Acknowledgements. The authors acknowledge the FESTIVAL team developed the browser for solar imaging data and the STEREO/SWAVES/SECCHI/ PLASTIC/MAG and GONG teams for their open data policy. We would like to thank the anonymous referee for their useful and constructive comments. R. Gómez-Herrero acknowledges the finnancial support by the Spanish Ministerio de Economía Competitividad (projects AYA2012-39810-820 C02-01 and ESP2013-48346-C2-1-R). The STEREO/SEPT project is supported under grant 50 OC 1302 by the German Bundesministerium für Wirtschaft through the Deutsches Zentrum für Luft- und Raumfahrt (DLR).

\section{References}

Acuña, M. H., Curtis, D., Scheifele, J. L., et al. 2008, Space Sci. Rev., 136, 203

Bougeret, J. L., Goetz, K., Kaiser, M. L., et al. 2008, Space Sci. Rev., 136, 487

Cane, H. V. 2003, ApJ, 598, 1403

Dresing, N., Gómez-Herrero, R., Klassen, A., et al. 2012, Sol. Phys., 281, 281

Ergun, R. E., Larson, D., Lin, R. P., et al. 1998, ApJ, 503, 435

Fitzenreiter, R. J., Fainberg, J., Weber, R. R., et al. 1977, Sol. Phys., 52, 477

Galvin, A. B., Kistler, L. M., Popecki, M. A., et al. 2008, Space Sci. Rev., 136, 437

Gómez-Herrero, R., Dresing, N., Klassen, A., et al. 2015, ApJ, 799, 55

Haggerty, D. K., \& Roelof, E. C. 2009, in AIP Conf. Ser. 1183, eds. X. Ao, \& G. Z. R. Burrows, 3

Howard, R. A., Moses, J. D., Vourlidas, A., et al. 2008, Space Sci. Rev., 136, 67 Ippolito, A., Pommois, P., Zimbardo, G., \& Veltri, P. 2005, A\&A, 438, 705

Kahler, S. W., Aurass, H., Mann, G., \& Klassen, A. 2007, ApJ, 656, 567

Kahler, S. W., Haggerty, D. K., \& Richardson, I. G. 2011, ApJ, 736, 106

Klassen, A., Aurass, H., Klein, K.-L., Hofmann, A., \& Mann, G. 1999, A\&A, 343,287

Klassen, A., Karlický, M., \& Mann, G. 2003, A\&A, 410, 307

Klassen, A., Gómez-Herrero, R., \& Heber, B. 2011, Sol. Phys., 1, 154

Klassen, A., Gómez-Herrero, R., Heber, B., et al. 2012, A\&A, 542, A28

Klein, K.-L., Krucker, S., Lointier, G., \& Kerdraon, A. 2008, A\&A, 486, 589

Lario, D., Raouafi, N. E., Kwon, R.-Y., et al. 2014, ApJ, 797, 8

Müller-Mellin, R., Böttcher, S., Falenski, J., et al. 2008, Space Sci. Rev., 136, 363

Ragot, B. R. 2006, ApJ, 653, 1493

Ragot, B. R., \& Kahler, S. W. 2008, Int. Cosmic Ray Conf., 1, 147

Raoult, A., \& Pick, M. 1980, A\&A, 87, 63

Richardson, I. G., von Rosenvinge, T. T., Cane, H. V., et al. 2014, Sol. Phys., 289,3059

Robbrecht, E., \& Berghmans, D. 2004, A\&A, 425, 1097

Strauss, R. D., \& Fichtner, H. 2015, ApJ, 801, 29

Tan, L. C., Reames, D. V., Ng, C. K., Saloniemi, O., \& Wang, L. 2009, ApJ, 701, 1753

Wang, L., Lin, R. P., Krucker, S., \& Gosling, J. T. 2006, Geophys. Res. Lett., 33, 3106 